\title{
A New Kolsky Bar Dynamic Spall Technique for Brittle Materials
}

\author{
A. W. Dean' ${ }^{1}$ W. F. Heard ${ }^{2}$ C. M. Loeffler ${ }^{3}$ B. E. Martin ${ }^{4} \cdot$ X. Nie $^{3}$
}

Received: 31 January 2016/ Accepted: 23 March 2016/Published online: 28 March 2016

(C) Society for Experimental Mechanics, Inc 2016

\begin{abstract}
For many years there have been controversial opinions on the value of dynamic tensile data obtained through the Kolsky bar spall tension technique. This is primarily due to the experimental conditions (i.e., specimen stress state and strain rate) not being well defined for these types of tests, thus making data interpretation and comparisons difficult. In this paper, a new spall theory is presented that ensures constant strain rate deformation while maintaining a uniform tensile stress within a large portion of the specimen. In light of this theoretical framework, pulse shaping was carefully designed to experimentally obtain this solution in Kolsky bar experiments. The incident wave generated under the guideline of the new theory has shown promising results for better refined Kolsky bar spall tension experiments on brittle materials.
\end{abstract}

Keywords Kolsky bar · Spall · Constant strain rate · Brittle materials

X. Nie

xnie@smu.edu

1 Mechanical and Energy Engineering, University of North Texas, Denton, TX, USA

2 U.S. Army Engineer Research and Development Center, Vicksburg, MS, USA

3 Mechanical Engineering, Southern Methodist University, Dallas, TX, USA

4 Air Force Research Laboratory, Munitions Directorate, Fort Walton Beach, FL, USA

\section{Introduction}

Dynamic tensile characterization of concrete materials has long been a challenging yet highly demanded research topic. The difficulties associated with this type of testing have mainly resulted from the extremely low tensile failure strength and small tensile failure strain, which are both inherent to this family of materials. Currently, the three most popular Kolsky bar-based dynamic tensile testing techniques for concrete are spall tension [1,2], split tension (Brazilian disk experiment) [3, 4], and direct tension [5, 6]. Each of these techniques has its own advantages and disadvantages. For the spall tension technique, the cylindrical specimens used in these types of experiments are usually directly cored from a concrete slab of interest, which makes them ideal representatives of the bulk material being investigated. However, the fundamental concepts for the Kolsky bar spall technique were largely inherited from the shock spall technique; therefore, the stress state and strain rate in the test specimens were not well defined compared to those in the Kolsky bar compression tests. In fact, both the stress and strain rates vary significantly along the length of the specimen during a spall test. This is also the main reason that, for many decades, spall tension data have not been considered as a direct comparison for dynamic tensile properties obtained through other relatively well-controlled dynamic testing techniques. In recent years, a few studies on concrete spall tension have shown the initial effort to maintain a constant strain rate deformation at the spall location of the specimen [7, 8]. These investigations indicated that relative constant strain rate deformation was realized at the failure location right before fracture occurred. Despite these initial findings, it is still unclear how (and why) the constant strain rate was realized. What's also unclear is whether it is possible to achieve both constant 
strain rate and uniform tensile stress over a majority of the spall specimen. Such experimental conditions, if proven to be feasible in spall tests, will revolutionize the Kolsky barbased dynamic spall technique not only for concrete but also for other brittle materials.

In this brief technical note, a new analytical approach was derived based on one-dimensional wave mechanics to explore the possibility of achieving constant strain rate deformation as well as uniform tensile stress state in a spall specimen. The feasibility of incorporating this theory to the Kolsky bar spall experiment was also demonstrated through careful pulse shaping. It is believed that novel experimental techniques built on this new spall theory will eventually help to bridge the gap in data interpretation between spall and other dynamic tensile testing techniques.

\section{Analysis}

Tensile stress in a spall specimen is generated when the incident compressive wave reflects back at the free surface of the specimen as a tensile wave. However, the resulting tensile stress profile in the specimen is determined by the overlap between both the incident and reflected waves. Figure 1 presents a general schematic of wave reflection/ overlap in a spall specimen with a linear elastic material response. The left-travelling reflected tensile stress wave, $\sigma_{1}=g(x, t)$, has the same profile and amplitude as the right-travelling incident compressive wave, $\sigma_{2}=f(x, t)$. At any location $x_{0}$ within the region where $f(x, t)$ and $g(x, t)$ overlap with each other, the stress in the specimen is given by:

$\sigma\left(x_{0}, t\right)=f\left(x_{0}, t\right)+g\left(x_{0}, t\right)$

While the stresses at two neighboring locations, $x_{0}+\Delta x$ and $x_{0}-\Delta x$, are:

$\sigma\left(x_{0}+\Delta x, t\right)=f\left(x_{0}+\Delta x, t\right)+g\left(x_{0}+\Delta x, t\right)$

$\sigma\left(x_{0}-\Delta x, t\right)=f\left(x_{0}-\Delta x, t\right)+g\left(x_{0}-\Delta x, t\right)$

The strain rate at location $x_{0}$, for any given time instance $t$, is defined by:

$\partial \varepsilon\left(x_{0}, t\right) / \partial t=\left(\partial g\left(x_{0}, t\right) / \partial t+\partial f\left(x_{0}, t\right) / \partial t\right) / E$

where $E$ is the Young's modulus of the specimen. The strain rates at $x_{0}+\Delta x$ and $x_{0}-\Delta x$ are:

$$
\begin{aligned}
\partial \varepsilon\left(x_{0}+\Delta x, t\right) / \partial t= & \left(\partial g\left(x_{0}+\Delta x, t\right) / \partial t\right. \\
& \left.+\partial f\left(x_{0}+\Delta x, t\right) / \partial t\right) / E \\
\partial \varepsilon\left(x_{0}-\Delta x, t\right) / \partial t= & \left(\partial g\left(x_{0}-\Delta x, t\right) / \partial t\right. \\
& \left.+\partial f\left(x_{0}-\Delta x, t\right) / \partial t\right) / E
\end{aligned}
$$

Similarly, the strain rate of location $x_{0}$ at time instances $t+\Delta t$ and $t-\Delta t$ are:

$$
\begin{aligned}
\partial \varepsilon\left(x_{0}, t+\Delta t\right) / \partial t= & \left(\partial g\left(x_{0}, t+\Delta t\right) / \partial t\right. \\
& \left.+\partial f\left(x_{0}, t+\Delta t\right) / \partial t\right) / E \\
\partial \varepsilon\left(x_{0}, t-\Delta t\right) / \partial t= & \left(\partial g\left(x_{0}, t-\Delta t\right) / \partial t\right. \\
& \left.+\partial f\left(x_{0}, t-\Delta t\right) / \partial t\right) / E
\end{aligned}
$$

Considering the respective travelling directions of the incident and reflective waves, and assuming $|\Delta x|=C_{0} \Delta t$ $\left(C_{0}\right.$ is the one-dimensional wave speed in the spall specimen), Eqs. (7) and (8) can be rewritten as:

$$
\begin{aligned}
\partial \varepsilon\left(x_{0}, t+\Delta t\right) / \partial t= & \left(\partial g\left(x_{0}+\Delta x, t\right) / \partial t\right. \\
& \left.+\partial f\left(x_{0}-\Delta x, t\right) / \partial t\right) / E \\
\partial \varepsilon\left(x_{0}, t-\Delta t\right) / \partial t= & \left(\partial g\left(x_{0}-\Delta x, t\right) / \partial t\right. \\
& \left.+\partial f\left(x_{0}+\Delta x, t\right) / \partial t\right) / E
\end{aligned}
$$

Fig. 1 Stress wave reflection and overlap in a spall specimen

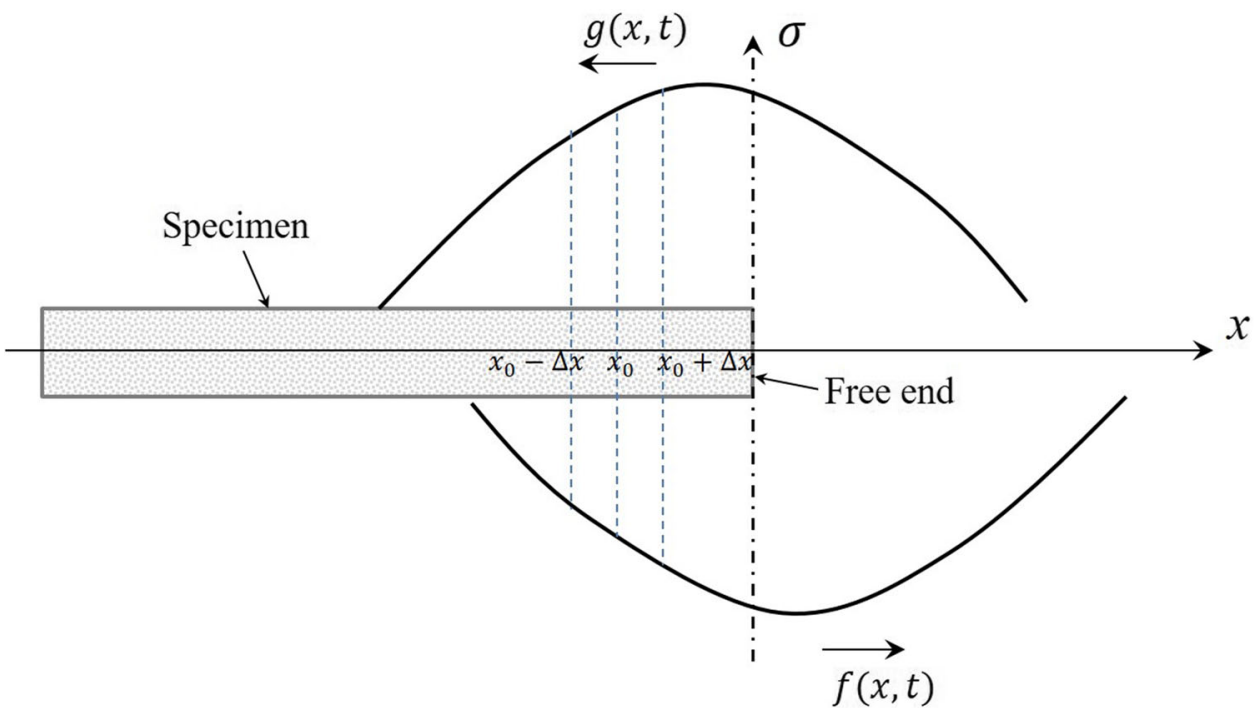


To satisfy the constant strain rate deformation criterion, the strain rate in the spall specimen should be independent of either location $(x)$ or time $(t)$. Therefore the following relation has to hold:

$$
\begin{aligned}
\partial \varepsilon\left(x_{0}+\Delta x, t\right) / \partial t & =\partial \varepsilon\left(x_{0}-\Delta x, t\right) / \partial t=\partial \varepsilon\left(x_{0}, t+\Delta t\right) / \partial t \\
& =\partial \varepsilon\left(x_{0}, t-\Delta t\right) / \partial t
\end{aligned}
$$

Using Eqs. (5), (6), (9), and (10) in light of Eq. (11) yields:

$\partial g\left(x_{0}+\Delta x, t\right) / \partial t=\partial g\left(x_{0}-\Delta x, t\right) / \partial t$

$\partial f\left(x_{0}+\Delta x, t\right) / \partial t=\partial f\left(x_{0}-\Delta x, t\right) / \partial t$

The only solutions for Eqs. (12) and (13) under any given $x_{0}$ and $\Delta x$ are $\partial g(x, t) / \partial t=C_{1}, \partial f(x, t) / \partial t=C_{2}$, where $C_{1}$ and $C_{2}$ are constants. This solution indicates that within the section of the specimen where the incident and reflected waves interact, both waveforms have to present linear stress profiles to maintain constant strain rate deformation. Under this condition, Eqs. (2) and (3) can be re-written as:

$\sigma\left(x_{0}+\Delta x, t\right)=f\left(x_{0}, t\right)-C_{2} \Delta t+g\left(x_{0}, t\right)+C_{1} \Delta t$

$\sigma\left(x_{0}-\Delta x, t\right)=f\left(x_{0}, t\right)+C_{2} \Delta t+g\left(x_{0}, t\right)-C_{1} \Delta t$

If the specimen is under uniform stress state, then:

$\sigma\left(x_{0}+\Delta x, t\right)=\sigma\left(x_{0}-\Delta x, t\right)$

Substituting Eqs. (14) and (15) into Eq. (16) leads to $C_{1}=C_{2}$. Note that $C_{1}$ and $C_{2}$ are the first order derivatives in time domain $(\sigma-t)$. To relate them to the stress slopes in the space domain $(\sigma-x)$ as shown in Fig. 1, one has to take into account that $g(x, t)$ is a left-travelling wave while $f(x, t)$ is a right-travelling wave. Therefore the following relation has to stand within the region of wave interaction where constant strain rate is maintained:

$\partial g(x, t) / \partial x=-\partial f(x, t) / \partial x$

The above analysis has shown that maintaining constant strain rate deformation and uniform stress in a spall specimen is theoretically possible if a portion of the incident and reflected waves carry a linear stress profile with opposite slopes in space domain. Considering that the reflected wave is actually the tensile version of the incident wave in Kolsky bar spall experiments, the incident wave itself essentially needs to carry the two linear stress profiles with opposite slopes. A possible experimental solution satisfying this requirement is to design an incident stress wave that resembles an isosceles triangle, as illustrated in Fig. 2. For this particular waveform, when the rising edge of the reflected wave fully enters the spall specimen and interacts with the trailing edge of the incident wave, a region with uniform tensile stress is generated (area between the dotted lines). As both waves propagate through the specimen, the amplitude of the uniform tensile stress increases until the brittle specimen fractures. Note that the isosceles triangular incident waveform is a special case of the solutions for Eqs. (12) and (13); therefore, the constant strain rate criterion is automatically satisfied.

\section{Experimental Feasibility Study}

Linear ramp incident waves have been previously used in Kolsky compression bar testing of various brittle materials [9-13]. In those dynamic uniaxial compressive experiments, ramp loading is essential to ensure constant strain rate deformation as well as dynamic stress equilibrium [14]. In our study, the challenge is to properly design the pulse shapers so that both the rising and trailing edges of the incident wave are linear ramps with slopes of the same amplitude. To test the feasibility of generating an isosceles triangular incident wave, a 19-mm diameter Kolsky compression bar setup was utilized for pulse shaping study with a $200-\mathrm{mm}$ long striker bar. The length of the incident bar was $3600-\mathrm{mm}$ and the strain gages were placed in the middle of bar. Through several iterations, it was found that compound pulse shapers fabricated from Teflon and annealed copper produced the best results. The dual material pulse shaper design presents a two-layer assembly, with a small Teflon disk $(3.175-\mathrm{mm}$ in diameter by 0.51-mm thick) stacking on top of an annealed copper disk (7.14-mm in diameter by $1-\mathrm{mm}$ thick). The incident wave generated by this type of pulse shaper is presented in Fig. 3. It is evident from Fig. 3 that the incident wave generally represents an isosceles triangular shape, which is desired for this novel spall technique. Closer examination of the waveform reveals that approximately $2 / 3$ of both the rising and trailing edges exhibit excellent linearity. The amplitude of the slopes of these two portions are within $\pm 3 \%$ variation. It is expected that once these two regions overlap during the spall test, the corresponding portion of the specimen shall experience constant strain rate deformation under a relatively uniform tensile stress state.

For further analysis, the incident wave and resulting reflected wave were hypothetically overlapped within an "imaginary" cylindrical concrete spall specimen with a length that fully covers the duration of the loading pulse. The evolution of the tensile strain profile resulting from this wave overlap is presented in Fig. 4. In Fig. 4, time "0" marks the onset of tensile strain in the specimen. After 5 $\mu \mathrm{s}$, the tensile strain becomes reasonably uniform across approximately $50 \%$ of the specimen's length. We assume that the Young's modulus of the concrete specimen is 50 
Fig. 2 Schematic illustrating how to create a uniform tensile stress in the spall specimen through the interaction of two isosceles triangular waves

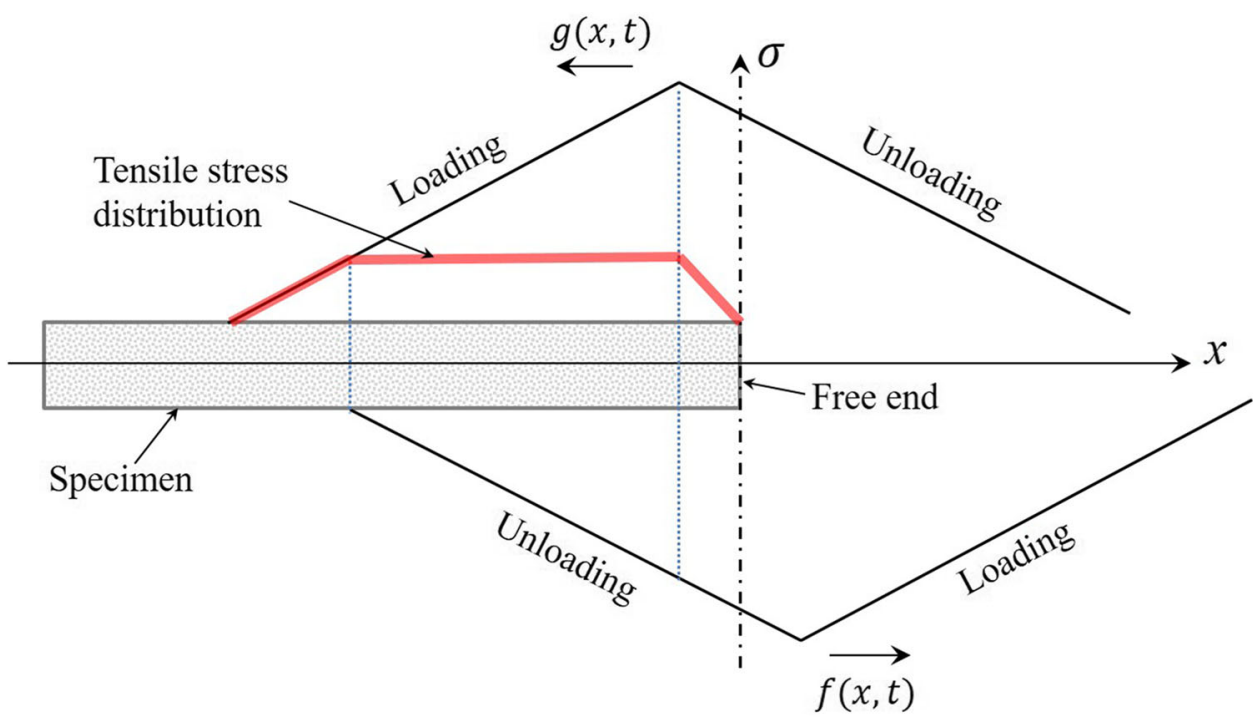

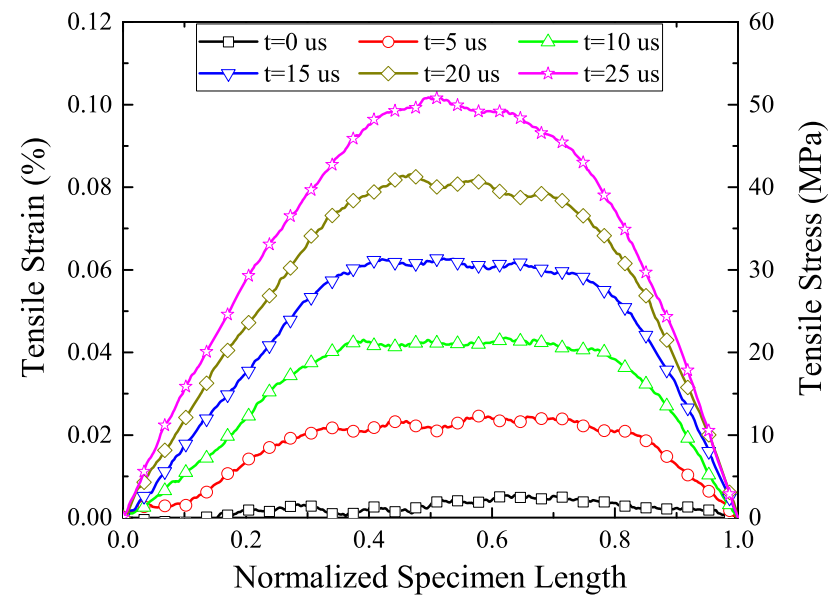

Fig. 4 Evolution of tensile strain distribution over the length of the spall specimen

specimen has reached a relatively uniform state before failure occurs. It needs to be noted that the wave dispersion effects may be negligible on our 19-mm diameter Kolsky bar especially with pulse shaping. For spall applications on larger diameter Kolsky bars, however, possible wave dispersion effects may impose complications to the data analysis and therefore need to be thoroughly considered and investigated before carrying out the stress analysis for the specimen. The tensile strain rate of the specimen is determined by the slope of the incident stress wave as indicated by Eq. (4). As a demonstration of achieving constant strain rate deformation under dynamic tension, the stress history profile at the center of the specimen is plotted in Fig. 5. Evidently, the stress increase shows an excellent linear relationship with time. For linear elastic materials, a constant stress rate loading is equivalent to constant strain 


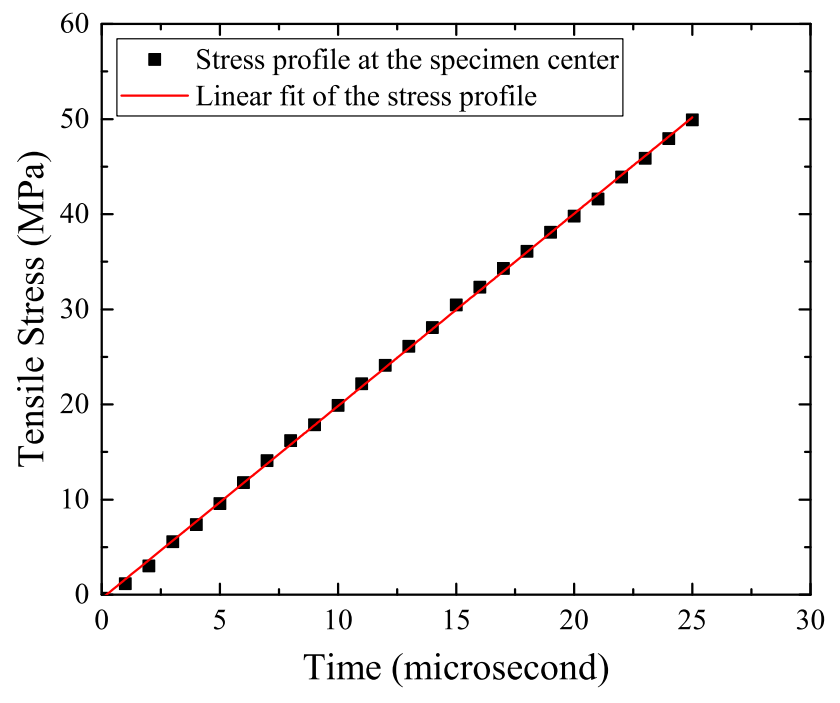

Fig. 5 The evolution of tensile stress versus time at the center of the spall specimen. The nearly constant stress rate indicates that the designed isosceles triangular incident wave is capable of achieving constant tensile strain rate loading in dynamic spall experiments

rate loading. The estimated tensile strain rate in this case study is around 40/s for a material with Young's modulus of $50 \mathrm{GPa}$.

\section{Summary}

This paper derives a new theoretical framework for a Kolsky bar-based dynamic spall technique. The derivation leads to the conclusion that, in order to maintain constant strain rate deformation as well as a uniform tensile stress state in the specimen, an incident stress wave resembling an isosceles triangle is required. The interaction of such an incident wave with its reflected counterpart gives rise to uniform tension over a considerable portion of the test specimen. In light of the analytical results, the feasibility to experimentally realize this special waveform was demonstrated through well-designed pulse shaping. This preliminary study lays the important foundation to a significant revolution for the dynamic spall technique, through which the spall tension results may be, for the very first time, directly compared with those obtained by other dynamic tensile testing techniques.

Acknowledgments Dr. Xu Nie is grateful for the financial support from the U.S. Army Engineer Research and Development Center through a sub-award from University of North Texas (Contract Number: W912HZ-14-C-0021). Permission to publish was granted by Director, Geotechnical and Structures Laboratory.

\section{References}

1. Klepaczko J, Brara A (2001) An experimental method for dynamic tensile testing of concrete by spalling. Int J Impact Eng 25:387-409

2. Wu H, Zhang Q, Huang F, Jin Q (2005) Experimental and numerical investigation on the dynamic tensile strength of concrete. Int J Impact Eng 32:605-617

3. Lambert D, Ross C (2000) Strain rate effects on dynamic fracture and strength. Int J Impact Eng 24:985-998

4. Gomez J, Shukla A, Sharma A (2001) Static and dynamic behavior of concrete and granite in tension with damage. Theor Appl Fract Mech 36:37-49

5. Ross C, Thompson P, Tedesco J (1989) Split-Hopkinson pressure-bar tests on concrete and mortar in tension and compression. ACI Mater J 86:475-481

6. Caverzan A, Cadoni E, Prisco M (2013) Dynamic tensile behavior of high performance fiber reinforced cementitious composites after high temperature exposure. Mech Mater 59:87-109

7. Erzar B, Forquin P (2010) An experimental method to determine the tensile strength of concrete at high rates of strain. Exp Mech 50:941-955

8. Forquin P, Erzar B (2010) Dynamic fragmentation process in concrete under impact and spalling tests. Int J Fract 163:193-215

9. Frew D, Forrestal M, Chen W (2001) A split Hopkinson bar technique to determine compressive stress-strain data for rock materials. Exp Mech 41:40-46

10. Frew D, Forrestal M, Chen W (2002) Pulse shaping techniques for testing brittle materials with a split Hopkinson pressure bar. Exp Mech 41:40-46

11. Jiao T, Li Y, Ramesh K, Wereszczak A (2004) high rate response and dynamic failure of structural ceramics. Int $\mathrm{J}$ Appl Ceram Technol 1:243-253

12. Nie X, Chen W, Sun X, Templeton D (2007) Dynamic failure of borosilicate glass under compression/shear loading experiments. J Am Ceram Soc 90:2556-2562

13. Heard W, Martin B, Nie X, Slawson T, Basu P (2014) Annular pulse shaping technique for large-diameter Kolsky bar experiments on concrete. Exp Mech 54:1343-1354

14. Chen W, Song B (2011) Split Hopkinson (Kolsky) bar: design, testing and applications. Springer, New York 